\title{
Adventures in Inflation And Cosmic Microwave Background - The future of the cosmos.
}

\author{
Rashmi Ramesh \\ SSN College of Engineering \\ Chennai, India \\ E-mail: johnsonrashmiegmail.com
}

The radiation present today as a $2.7 \mathrm{~K}$ thermal background originated when the universe was denser by a factor of $10^{9}$ and younger by a factor of around $5 \times 10^{4}$. The radiation provides the most distant direct image of the universe we can hope to see, at least until gravitational radiation becomes a useful astronomical data source. The microwave background radiation is extremely uniform, varying in temperature by only a few parts in $10^{5}$ over the sky (apart from an overall dipole variation arising from our peculiar motion through the microwave background's rest frame); its departure from a perfect blackbody spectrum has yet to be detected.

The 34th International Cosmic Ray Conference

30 July- 6 August, 2015

The Hague, The Netherlands

\section{- Introduction}

About 380,000 years after the Big Bang, the temperature of the Universe had dropped sufficiently for electrons and protons to combine into hydrogen atoms, $\mathrm{p}+\mathrm{e} \square \mathrm{H}$. From this time onwards, cosmic radiation was effectively unable to interact with the background gas; it has propagated freely ever since, while constantly losing energy because its wavelength is stretched by the expansion of the Universe. 
Originally, the radiation temperature was about 3000 degrees Kelvin, whereas today it has fallen to only $3 \mathrm{~K}$.

Observers detecting this radiation today are able to see the Universe at a very early stage on what is known as the 'surface of last scattering'. Photons in the cosmic microwave background have been travelling towards us for over ten billion years, and have covered a distance of about a million billion billion miles.

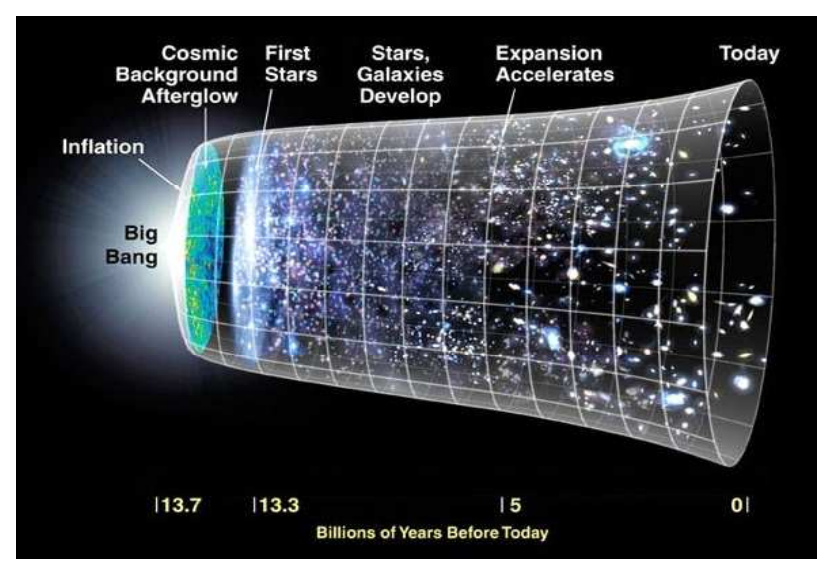

\section{- The Temperature of the universe today}

Measurements of the cosmic microwave background radiation (CMB) allow us to determine the temperature of the Universe today. The brightness of the relic radiation is measured as a function of the radio frequency. It is approximately described by thermal radiation distributed throughout the Universe with a temperature of about 2.735 degrees above absolute zero. This is a dramatic and direct confirmation of one of the predictions of the Hot Big Bang model.

The Cosmic Background Explorer (COBE) satellite measured the spectrum of the cosmic microwave background in 1990, showing remarkable agreement between theory and experiment. The diagram to the right shows the results plotted in waves per centimeter versus intensity. The theoretical best fit curve (the solid line) is indistinguishable from the experimental data points (the point-size is greater than the experimental errors). 


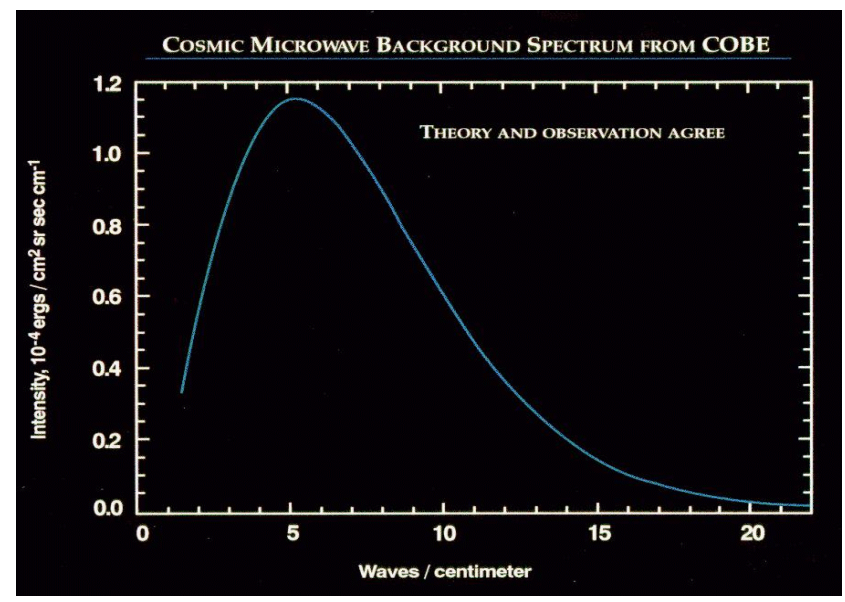

\section{- $\quad$ Ripples At The Edge Of The Universe}

Suspendisse lectus leo, consectetur in tempor sit amet, placerat quis neque. The COBE satellite was launched twenty five years after the discovery of the microwave background radiation in 1964. In spectacular fashion in 1992, the COBE team announces that they had discovered 'ripples at the edge of the Universe', that is, the first sign of primordial fluctuations at 380,000 years after the Big Bang. These are the imprint of the seeds of galaxy formation.

These appear as temperature variations on the full sky picture that COBE obtained. They are at the level of only one part in one hundred thousand. Viewed in reverse the Universe is highly uniform in every direction lending strong support for the cosmological principle.

The thumbprint image below shows the temperature of the Universe in all directions projected onto a plane (similar to a map of the earth):

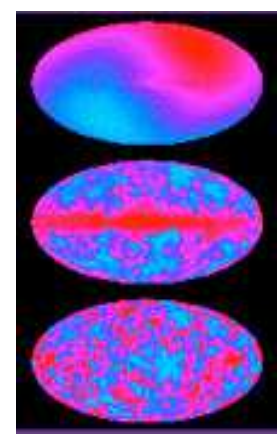

1. The raw temperature map (top) has a large diagonal asymmetry due to our motion with respect to the cosmic microwave background - a Doppler shift.

2. The temperature fluctuations after subtraction of the velocity contribution, showing primordial fluctuations and a large radio signal from nearby sources in our own galaxy (the horizontal strip). 
3. The primordial fluctuations after subtraction of the galaxy signal.

\section{- The Origin Of Fluctuations In The CMB}

There are a variety of competing theories for the origin of large-scale structure and the fluctuations in the cosmic microwave background radiation. These models fall into two categories: (1) causal topological defect models, such as cosmic strings and textures, or (2) inflationary scenarios.

Examples of the different cosmic microwave fluctuation patterns that they create are shown below for maps of a few degrees. The random features of inflationary models and cosmic strings are quite similar, being quite distinct from the stronger features for other defects. Experimental improvements in the study of such small scale CMB fluctuations should lead to discriminating tests between these different models.
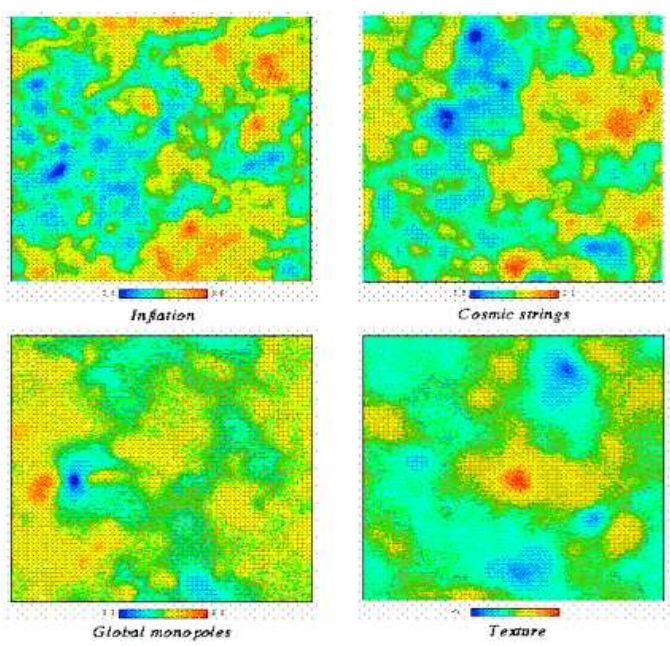

\section{- The Inflatory Universe}

According to the theory of inflation, the early Universe expanded exponentially fast for a fraction of a second after the Big Bang. Cosmologists introduced this idea in 1981 to solve several important problems in cosmology. One of these problems is the horizon problem. Assume, for a moment, the Universe is not expanding. Now imagine a photo was released very early in the Universe and travelled freely until it hits the North Pole of the Earth. Now imagine another photo was released at the same time, but "opposite" to the first one. It will hit the Earth at the South Pole. Could these two photons exchange any information from the time when they are released? Clearly not, because the time required to send information from one photo to the other would be two times the age of the Universe. The photons are causally disconnected. They are outside of each other's horizon. 


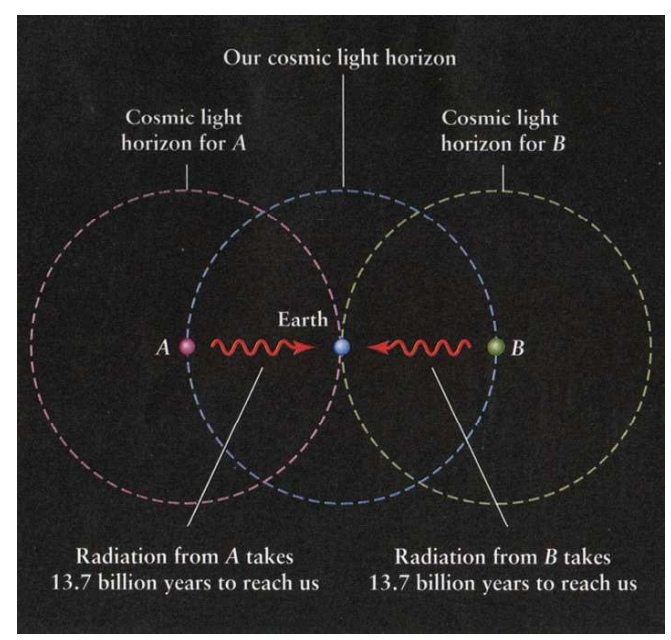

These photons could not have communicated with each other unless inflation took place during the very early Universe.

However, we observe that photons from opposite directions must have communicated somehow, because the cosmic microwave background radiation has almost exactly the same temperature in all directions over the sky.

This problem can be solved by the idea that the Universe expanded exponentially for a short time period after the Big Bang. Before this period of inflation, the entire Universe could have been in causal contact and equilibrate to a common temperature. Widely separated regions today were actually very close together in the early Universe, explaining why photons from these regions have (almost exactly) the same temperature.

A simple model for the expansion of the Universe is to consider the inflation of the balloon. A person at any point on the balloon might consider themselves to be at the centre of the expansion, as all neighbouring points are getting further away. During inflation, the Universe expanded by a factor of about $\mathrm{e}^{60}=10^{26}$. This number is a one followed by 26 zeros. It transcends normal political/economic discussions of inflation.

\section{- Quantum Fluctuations}

We are able to observe quantum fluctuations that were created at the beginning of inflation. The expansion of the Universe during the inflationary epoch serves as a huge microscope that magnifies quantum fluctuations, corresponding to a scale less than $10^{-28} \mathrm{~cm}$, to cosmological distances. This leaves imprints in the cosmic microwave background radiation (hotter and colder regions) and in the distribution of galaxies 


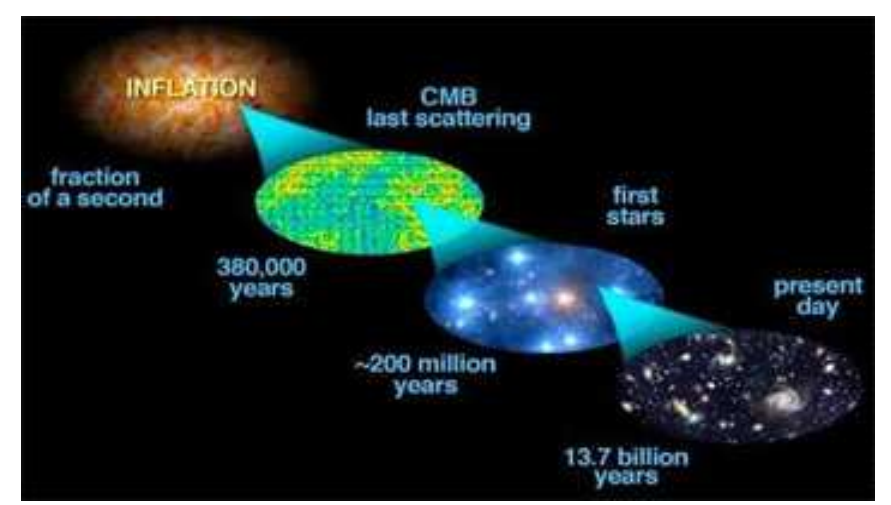

Inflation works as a cosmic microscope to see the quantum fluctuations in the very early Universe

Using classical physics, the evolution of the inflationary Universe is homogeneous - each spatial point evolves exactly the same way. However, quantum physics introduces some uncertainty in the initial conditions for the different spatial points.

These variations act as seeds for structure formation. After the inflationary period, when fluctuations are amplified, the density of matter will vary slightly from place to place in the Universe. Gravity will cause the more dense regions to start contracting, leading to the formation of galaxies.

\section{- How Much Matter Does The Universe Contain?}

An important question today in cosmology is how much mass is contained in the Universe. If there were no matter filling the Universe, the Universe would expand forever and the recession velocity of objects at rest with respect to the expansion of the Universe would not change as the Universe expands. We know, of course, that the Universe is not empty but filled with matter, and ordinary matter through gravity attracts other matter, causing the expansion of the Universe to slow down. If the density of the Universe exceeds a certain threshold known as the critical density, this gravitational attraction is strong enough to stop and later reverse the expansion of the Universe, causing it eventually to recollapse in what is known as the "Big Crunch." On the other hand, if the average density of the Universe falls short of the critical density, the Universe expands forever, and after a certain point the expansion proceeds much as if the Universe were empty. A critical Universe lies precariously balanced between these two possibilities. 


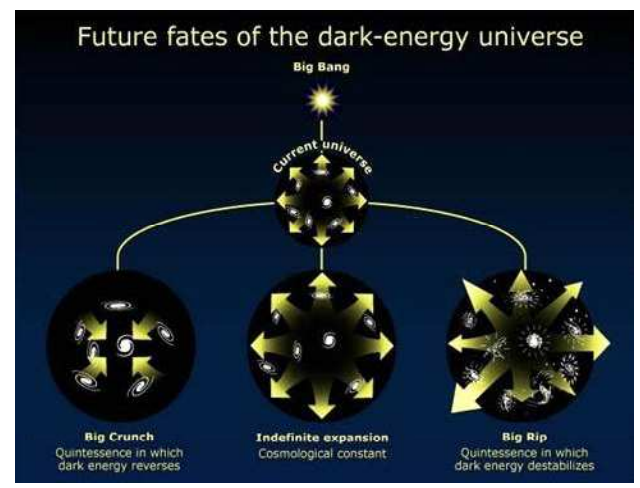

\section{- $\quad$ Big Rip, Big Freeze, Or Big Crunch : Possible End States Of The Univerrse}

Measurements of the cosmic microwave background radiation

According to the

Friedmann equation of energy, the future of the expansion speed of the universe might then become a competition between the term that describes the evolution of this energy form and the trailing curvature term. Curvature will not affect the magnitude of the acceleration of the expansion.

Let's first look at energy forms that dilute slower than $\mathrm{a}^{-2}$. These have $\omega<-1 / 3$ and will dominate the curvature term, which goes as $\mathrm{a}^{-2}$. In these universes it does not matter what total and the curvature is at any time - the universe will always expand and qualitatively act as the $\Omega=1$ universe described earlier, ending either in a Big Rip or a Big Freeze.

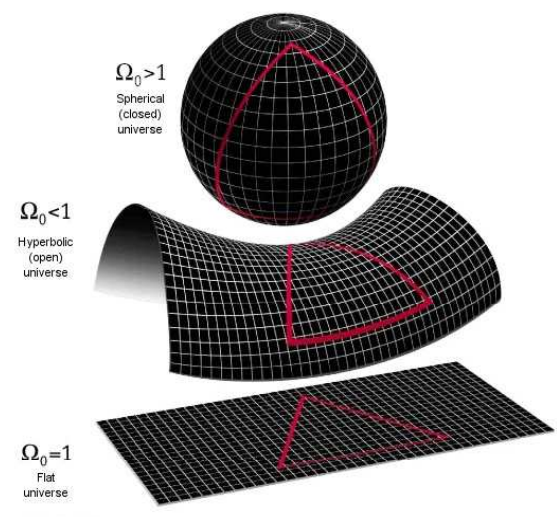

For a universe dominated by cosmic strings, there is a tie between the energy term and the curvature term - both go as $\mathrm{a}^{-2}$. Qualitatively this universe will evolve as a $=1$ universe with no curvature, ending in a Big Freeze. For a universe dominated by matter, radiation, or ultralight, a finite curvature term will eventually grow to dominate any of these terms as the universe expands. If $\left(1-\Omega_{\text {total }}\right)$ $>0$, then $\Omega_{\text {total }}<1$, and the universe will expand forever and end in a Big Freeze. This case is called an "open universe." If $\left(1-\Omega_{\text {total }}\right)<0$, then $\Omega_{\text {total }}>1$, and eventually the expansion rate 'a will go to zero. At that time a maximum expansion scale factor of the universe can be found. Past this time, these universes 
will collapse into a Big Crunch. It is also useful to understand that any universe that spends a significant amount of time being dominated by a $\omega<-1 / 3$ energy phase drives $\Omega_{\text {total }}$ to unity. Alternatively, a universe that spends a significant time being dominated by a $\omega>-1 / 3$ phase drives total away from unity.

If present observations continue to support the concordance cosmology where roughly $70 \%$ of the energy in the universe is in a form where $\omega=-1$, and if the energy forms in the universe today are stable, then the universe should continue to expand and the average $\omega$ of the universe will continue to approach -1 . The fate of the universe will then be a Big Freeze. It is possible that the $\omega=-1$ energy will evolve as a field and/or decay into energy forms with higher $\omega$. If so, the ultimate future of the universe is unknown.

\section{- $\quad$ Finale : Testing Inflationary Cosmology}

"The universe is not only queerer than we suppose,

It is queerer than we can suppose."

-J.B.S. Haldane.

The cosmic microwave background radiation is a remarkably interesting and powerful source of information about cosmology. It provides an image of the universe at an early time when the relevant physical processes were all very simple, so the dependence of anisotropies on the cosmological model can be calculated with high precision. At the same time, the universe at decoupling was an interesting enough place that small differences in cosmology will produce measurable differences in the anisotropies. The microwave background has the ultimate potential to determine fundamental cosmological parameters describing the universe with percent-level precision. If this promise is realized, the standard model of cosmology would compare with the standard model of particle physics in terms of physical scope, explanatory power, and detail of confirmation.

\section{References}

- $\quad$ Michio Kaku, Parallel Worlds, 01

- $\quad$ Stephen Hawking, A briefer History of Time, Penguin Editions, 3018.

- Arnold Penzias, The Origin Of Elements, in Nobel Lectures Physics 1971-1980, Editor Stig Lundqvist, World Scientific Publishing Co., Singapore, 1992. 